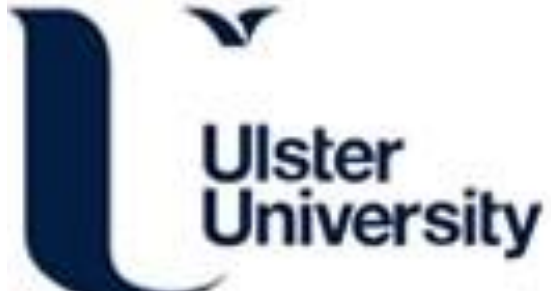

An loT Framework for detecting Movement within Indoor Environments.

Curran, K., Mansell, G., \& Curran, J. (2018). An loT Framework for detecting Movement within Indoor Environments. In International Conference on Machine Learning for Networking (MLN'2018)

Link to publication record in Ulster University Research Portal

\section{Published in:}

International Conference on Machine Learning for Networking (MLN'2018)

Publication Status:

Published (in print/issue): 30/11/2018

\section{Document Version}

Author Accepted version

\section{General rights}

Copyright for the publications made accessible via Ulster University's Research Portal is retained by the author(s) and / or other copyright owners and it is a condition of accessing these publications that users recognise and abide by the legal requirements associated with these rights.

\section{Take down policy}

The Research Portal is Ulster University's institutional repository that provides access to Ulster's research outputs. Every effort has been made to ensure that content in the Research Portal does not infringe any person's rights, or applicable UK laws. If you discover content in the Research Portal that you believe breaches copyright or violates any law, please contact pure-support@ulster.ac.uk. 


\title{
An IoT Framework for detecting Movement within Indoor Environments
}

\author{
Kevin Curran ${ }^{1}$, Gary Mansell ${ }^{1}$, Jack Curran ${ }^{2}$ \\ ${ }^{1}$ Ulster University, School of Computing and Intelligent Systems \\ Londonderry, Northern Ireland \\ Kj.curraneulster.ac.uk \\ ${ }^{2}$ Open University, Walton Hall, Milton Keynes, UK
}

\begin{abstract}
Tracking people indoors can be valuable in smart living scenarios such as tracking shoppers in a mall or in healthcare situations when tracking the movement of elderly patients can allow them to remain more independent. Determining accurate movement of people indoors is problematic however as there is no universal tracking system such as GPS which works indoors. Instead, a range of techniques are used based on technologies such as cameras, radio frequency identification, $\mathrm{WiFi}$, Bluetooth, pressure pads and radar are used to track people and objects within indoor environments. The most common technologies for tracking are Bluetooth and WiFi. Many Internet of Things (IoT) devices support these protocols and can therefore act as beacons and hubs for movement detection indoor. We provide here an overview of an IoT focused framework which allows the plug and play of Bluetooth and WiFi devices in addition to integrating passive and active approaches to determining the movement of people indoors.
\end{abstract}

Keywords: Movement Detection, IoT, Device Free Passive Localisation, Presence Detection

\section{Introduction}

There is a substantial amount of work in determining the location or activity of an individual over time inside a building with wireless tracking [1, 2]. Movement detection is important for many smart living scenarios such as health care, games, manufacturing, logistics, shopping, security and tour guides. Indoor localisation systems can be classified into active and passive systems. Using Wireless signals is an attractive and reasonably affordable option to deal with the currently unsolved problem of widespread tracking in an indoor environment. A subset of tracking people is with context aware solutions. Context aware computing applications that adapts to their location of use, the collection of nearby people or objects, as well as changes to those objects over time. Location-aware services (LAS) are special context-aware application that recommend suitable services to a user based on the user's location. They enable location intelligence which provides many benefits such as personalisation of marketing communications, consumer analytics, locating a fireman in a burning building or concluding the optimal place for a group to meet for a common activity. Indoor location determination has become a crucial component in many applications and a standard for indoor localisation does not exist yet. LAS is therefore an important aspect for the Internet of Things (IoT). Current implementations of location intelligence (via LAS) in a mobile environment suffer from several issues and the choice of which technology to make use of is critical. Location tracking techniques can be classified into two broad categories; active 
localisation and passive localisation. The distinguishing factor is the participation of the tracked individual. In a passive system, the user is not required to participate, i.e. the system can track them without any need for an electronic device to be carried or attached which sends out signals to help deduce their location. In an active system, an electronic device is carried. Device Free Passive Localisation (DfPL) approaches can identify human presence by monitoring variances of the signal strength in wireless networks [3]. This is since human body contains about $70 \%$ water and it is known that waters resonance frequency is $2.4 \mathrm{GHz}$. An indoor wireless positioning system consists of at least two separate hardware components: a signal transmitter and a measuring unit. The latter usually carries the major part of the system intelligence. Mobile devices face many challenges that are intrinsic to mobility and are unlikely to be overcome easily. Wireless connectivity is highly variable and mobile devices must rely on limited energy sources. These issues often significantly hinder implementations of mobile LAS. The core concepts used to locate an object are explained in the next section which is then followed by a review of current location detection technologies which may be used to implement a Location based system $[4,5]$.

\section{Detecting User Movement Indoors}

A significant drawback of many indoor locating technologies is the requirement to deploy a costly and complex infrastructure composed of dedicated hardware. The existing IEEE 802.11 networks and support for wireless protocols by the clear majority of mobile devices makes Wi-Fi a logical choice for low-cost indoor location detection. Wireless networks are capable of tracking movement through the network using a technique known as radio mapping or more commonly fingerprinting, which most IEEE 802.11 based location detection approaches are based on. Fingerprinting requires a complex setup or training phase to construct a map of pre-recorded received signal strengths (RSS) from nearby access points (AP's) at every position of an interesting area. The results are stored in a fingerprint database which can be queried with any RSS to identify and map corresponding locations. This fingerprint database or radio map can be used to create a model such as the example shown in Figure 1.

Fingerprinting provides good accuracy but is highly vulnerable to environmental changes such as rearranging furniture or moving the APs. One method of reducing this factor is collaborative feedback allowing a continually evolving radio map however the variation in RSS generated by different Wi-Fi chips could be a significant limitation in using a Wi-Fi based approach. An important consideration is that the decisions made when installing a $\mathrm{Wi}-\mathrm{Fi}$ AP were typically to catch large congregations of users and primarily to provide the highest available throughput to those users. Indoor environments are also especially noisy with other wireless devices such as wireless headsets and cordless phones causing unpredictable interference. These factors result in a coverage area which is less than ideal for fingerprinting. Fingerprinting can be divided into deterministic and probabilistic approaches. There are many models within each category each with their own pros and cons, overall the probabilistic models are the most promising with the most notable being the Bayesian-Hidden Markov model. This model provides accuracy of 1.5 meters but lacks reliability with only $70 \%$ of 
results being correct. It is also worth noting that depending on location, Wi-Fi based methods may be impossible or even dangerous to consider, for instance use in a hospital could be dangerous as the Wi-Fi signals may interfere with hospital equipment signals.

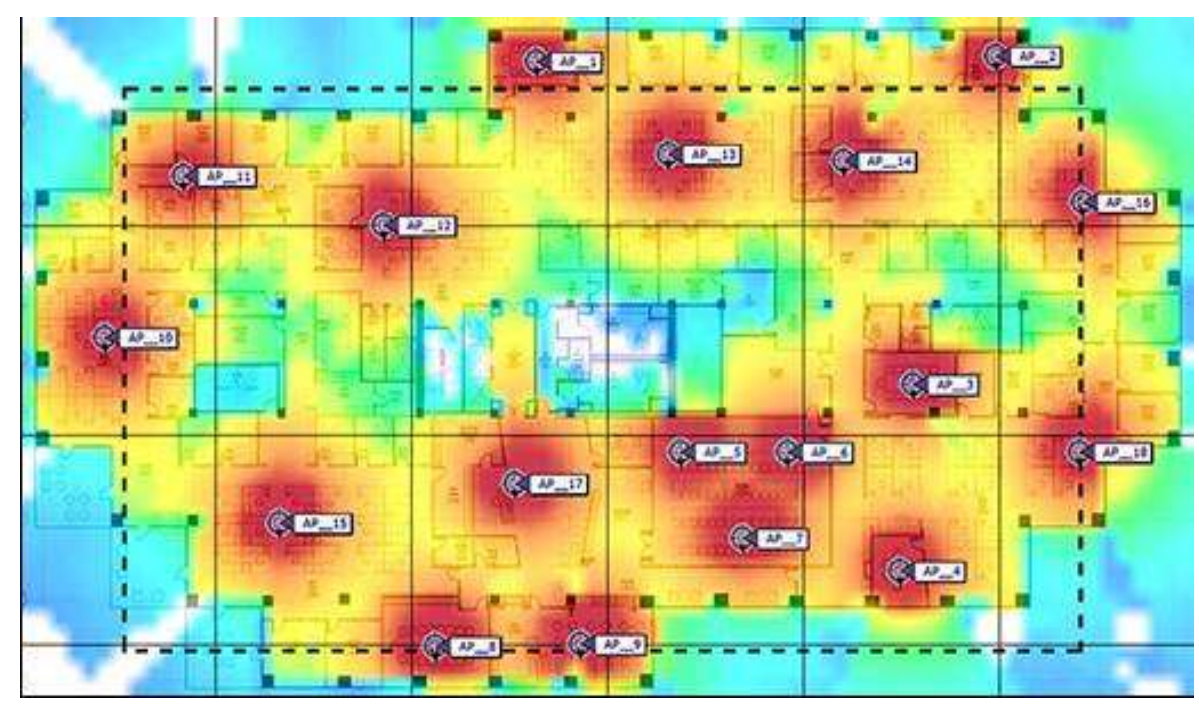

Figure 1: Radio map

Received Signal Strength Indicator (RSSI) is the most crucial parameter in the localization of WLAN devices [6]. At the monitoring device e.g. laptop, phone, it shows the signal strength received from an access point, where the stronger signal received by the WLAN card, the closer the position of the card to the access point. One of the most important factors in the measurement of RSSI is the power attenuation due to distance; however, absorption gradient also affects the RSSI measurement. Sudden changes in signal absorption, due to walls for example, introduce discontinuities into the dependence between RSSI and distance which is normally considered a smooth function [7]. In addition to walls, the presence of humans, the thickness of walls and doors, the air temperature, nearby devices, the direction of the antenna, and the types of WLAN cards influence the absorption of the RF signal energy. The RSSI values can be reported by the device driver as a non-dimensional number or percentage and sometimes is converted to $\mathrm{dBm}$ through some nonlinear mapping process [8]. The error caused by different types of WLAN cards can be around $20 \%$ which is a considerable error value [9].

\section{IOT Movement Detection Framework}

This section provides an overview of an indoor tracking architecture including the web application and the mobile app which we have developed which builds on our previous work $[10,11,12,13]$. This IoT focused framework allows the use of different indoor tracking technologies to be plugged in and it also allows the use of passive and active 
tracking technologies. The web application interacts with the system by a range of different types of clients. It written in Java. The web application offers content in a RESTful way. Access to the web portal is via JSP pages which are compiled into HTML mark-up with Ajax used as the primary means of transferring data. Data transfer is based on the commonly used JSON data interchange format so as to permit reuse of functionality by the mobile application. Figure 2 shows the communication procedure with the web application. The request and response may or may not contain JSON data.

\section{Application Communication}

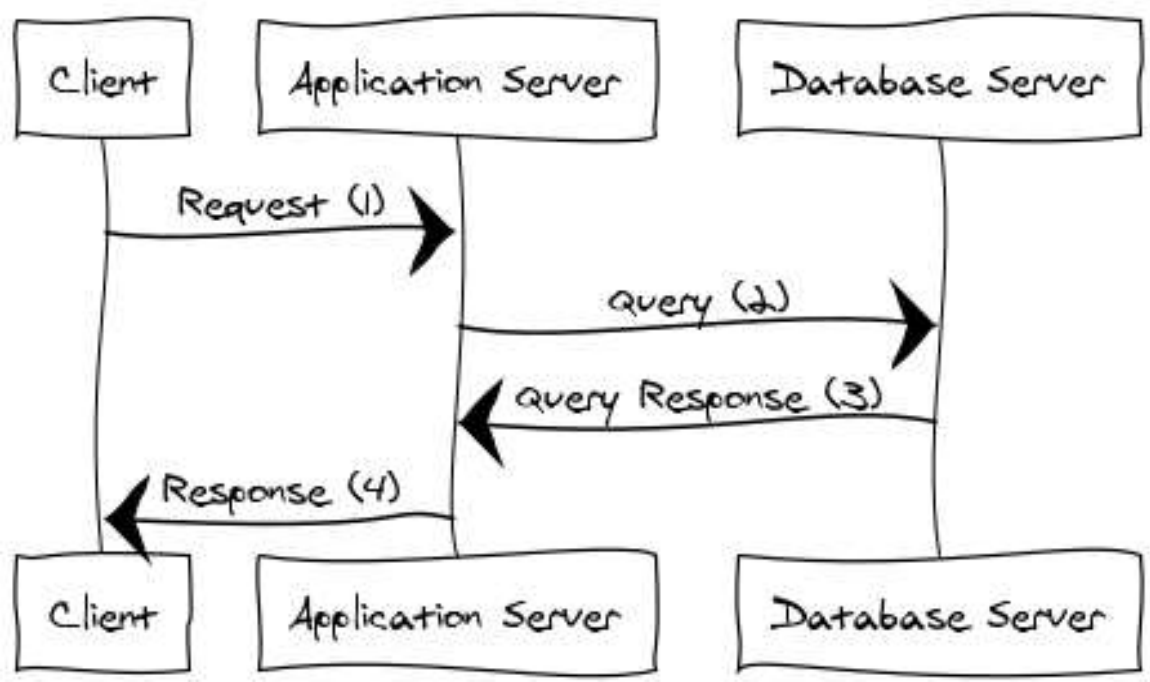

Figure 2: Web app communication

The web application is modular and loosely coupled allowing functionality to easily be reused or replaced entirely if required. This allows us to create a framework where location sensors can be ibeacons or Eddystone beacons. They could also be another technology such as RFID or Zigbee. The framework makes it simple to slot in the location determination technology of choice. A three-tiered system is used to achieve this where three distinct tiers are made up from a data access layer, a business layer and a presentation layer. Each layer has clearly defined roles. The data access layer is used for communication with the database only. The business layer stores application specific logic. The presentation layer contains user interaction logic as well as offering an endpoint for client communication. It also communicates with the business layer and the business layer communicates with the data access layer which in turn communicates with the database. We took time to design the system like this so that future IoT location determination technologies can be more easily plugged in. 


\subsection{Mobile Application}

The mobile application uses the Android beacon library to communicate with nearby beacons to retrieve their namespace id and instance ids. The Android beacon library is an open source library with a large support base and is regularly updated for bug fixes and support for new features is added regularly. Once retrieved, the Eddystone-UID communicates with the server and this determines the user's current position. The mobile app is used by most of the system users. The initial start page of the mobile application allows users to login with the username/password combination if they already registered. If no registration has been done previously, then new users can navigate to the registration page to create a new account.

Beacon scanning allows a user to enable scanning for beacons with a prompt shown if Bluetooth is not already enabled. Once scanning is enabled, the scanning functionality of the mobile application lets beacons be scanned for every ten seconds. If a beacon (closer than the ranging distance threshold) is found, then its Eddystone-UID details are sent to the web application which saves a new position history with the current timestamp. While remaining within range of the same beacon, new position histories are saved every minute. If multiple beacons are scanned, then the closest beacon is used. Once beacon scanning is enabled it will continue to run in the background even if the user closes the application completely. We choose this because beacon scanning is performed in a custom background service which the application communicates with. This becomes problematic however when attempting to get the current status of the background service after restarting the application as Android does not provide a clear way of checking if a service is running or not. Nevertheless, if the application does restart, the state of the background service is correctly shown by the state of the buttons. Although the normal scan cooldown time results in very little additional battery consumption, a power saving mode has been included which increases the scanning cooldown to thirty seconds. This shortens the time it takes to discover new beacons although user locations are still saved often enough to be considered accurate.

The position history page allows a user to view all their own position histories in a list. When a position history is selected, specific details are shown including the time and date they visited each position. This page automatically updates each time a new position history is saved (if beacon scanning is enabled) allowing the user to see in real time which location they are in. The account settings page allows users to manage their account details such as name and password. The user account id and username are also shown (to use if admin support is required) and validation errors result in icons like those on the login and registration pages. The URL that the application uses can be changed here. The reset button allows a user to reset any changes they have made.

The manage user accounts page displays all information relating to existing user accounts such as usernames, user account types and full names. From this part, an administrative user can create, update or delete user accounts.

To assign beacons to positions, there must be locations that the positions are associated with. The manage locations page allows administrative users to manage locations 
including the ability to create, update and delete location data. Deleting a location also deletes any positions which are assigned to that location, with the user made aware of this while confirming deletion. There is no limit on the number of locations however some manufacturers do have limits on the number of beacons assigned to a network.

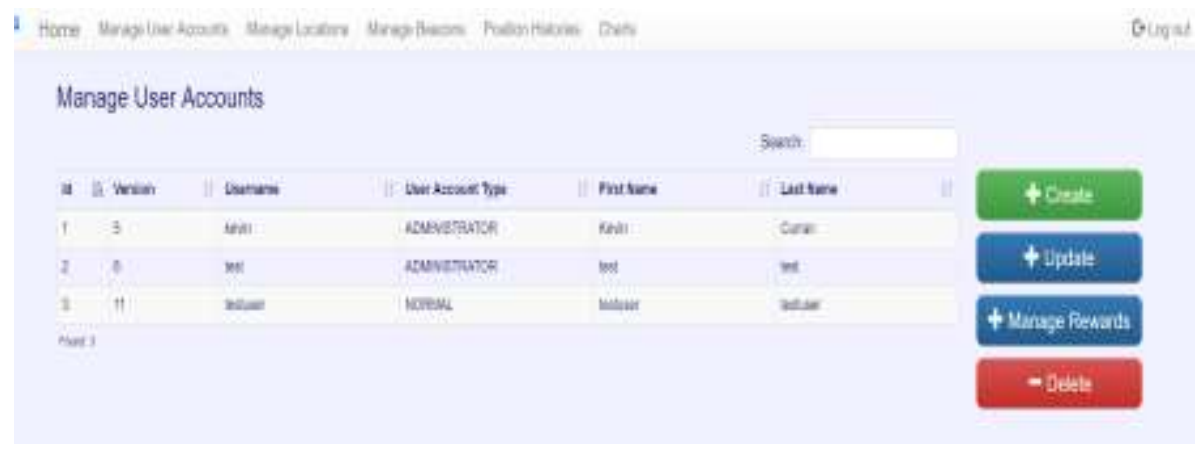

Figure 3: Manage user accounts page

Managing beacons is one of the key features of the web portal and the manage beacon page allows administrators perform this. Beacons can be created, modified and deleted as shown in Figure 4. During creation or modification, beacons can be optionally assigned to a location via a dropdown which is populated with locations saved through the manage locations page. If a beacon is assigned a location, then a position name must be entered.

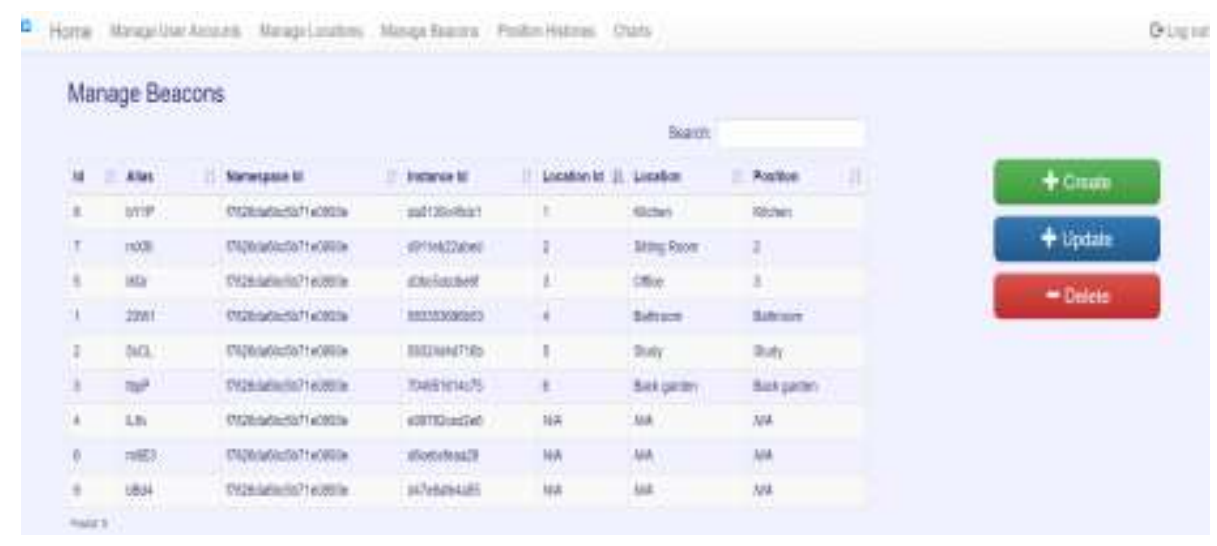

Figure 4: Manage beacons page

The view position histories page can be used by admins to view position histories for all users within the system (see Figure 5). The admin can also elect to auto refresh this 
page meaning table data will automatically update every few seconds, displaying any new position histories which may have been saved.

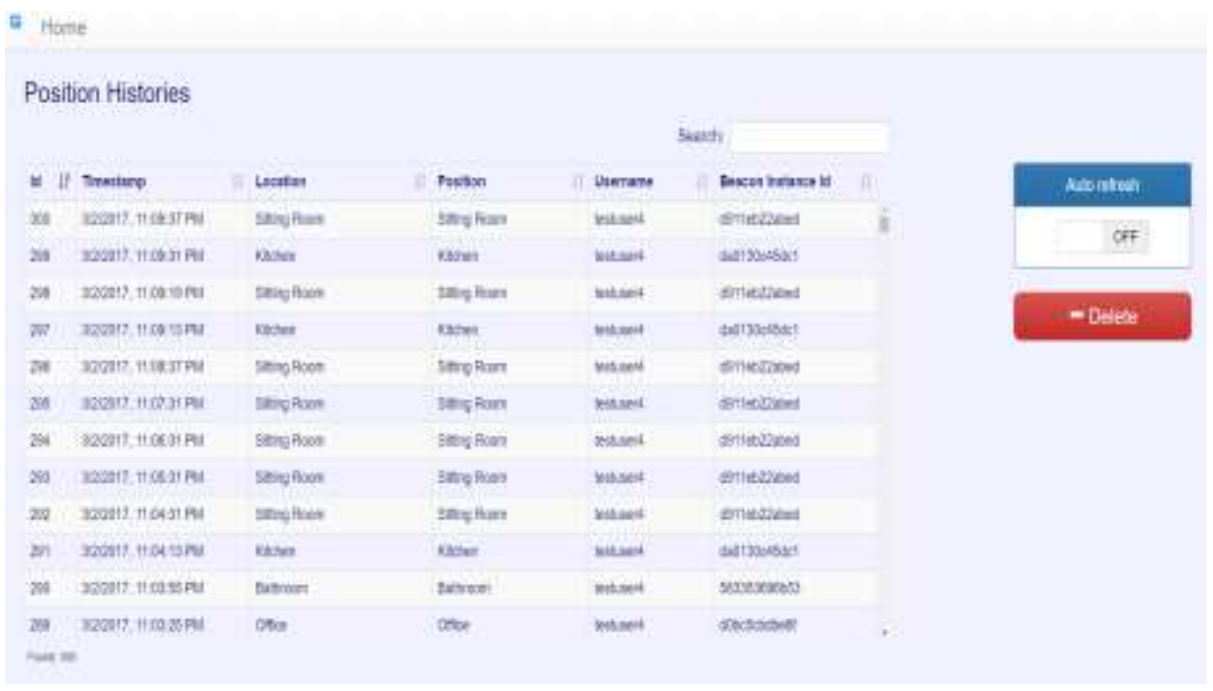

Figure 5: View position histories page

Viewing charts allows an admin user to see various statistics about the system including the most popular locations by total linger time, most visited positions for a location by location linger time, average linger time for a location, and the overall top system users by reward points. Linger time and session times are calculated based on the amount of continuous time each user spends at a position, referred to as a position history session. All position history sessions are first determined to produce the chart data displayed on this page. This section also has auto refresh functionality which can be enabled or disabled to view a snapshot or real time statistics for the system whereby all chart data automatically updates as changes happen on the network when beacons send back updates. An administrative user can hover over each chart which displays additional detail about the targeted data in a popup. A user can select locations on this chart which updates the other charts with data specifically for the chosen location.

\section{Conclusion}

We presented an overview of an extensible indoor location determination framework. It utilizes Bluetooth beacons for active positioning which can determine location of individuals. These beacons can use both ibeacon or Eddystone standards. The framework also facilitates device free passive localisation techniques for determination of activities performed in each location. The Bluetooth active localisation hardware can easily be substituted with another active technology such as WiFi. The framework 
Please cite as: Kevin Curran, Gary Mansell, Jack Curran (2018) An loT Framework for detecting Movement within Indoor Environments. International Conference on Machine Learning for Networking (MLN'2018), Paris, France, November 27-29, 2018

allows the easy updating of new locations, beacons and activities which are all important aspects of the future Internet of Things.

\section{References}

1. Vance, P., Prasad, G., Harkin, J., Curran, K. (2010) Analysis of Device-free Localisation (DFL) Techniques for Indoor Environments. ISSC 2010 - The 21st Irish Signals and Systems Conference, University College Cork, 23rd - 24th June

2. Vance, P., Prasad, G., Harkin, J., Curran, K. (2011) A wireless approach to Device-Free Localisation (DFL) for indoor environments. Assisted Living 2011 - IET Assisted Living Conference 2011, IET London: Savoy Place, UK, 6 April 2011

3. Deak, G., Curran, K., Condell, J. (2010) Evaluation of Smoothing Algorithms for a RSSI-based Device-free Passive Localisation. Advances in Intelligent and Soft Computing, pp: 59-66, Springer-Verlag 2010, ISBN: 978-3-642-162947, DOI 10.1007/978-3-642-162-16295-4

4. Furey, E., Curran, K., Lunney, T., Woods, D., Santos, K (2008a). Location Awareness Trials at the University of Ulster, Networkshop 2008 - The JANET UK International Workshop on Networking, The University of Strathclyde, 8th-10th April 2008

5. Bekris, K., Rudys, A., Marceau, G., Kavraki, L., Wallach, D. (2002) Robotics based location sensing using wireless Ethernet, The Eighth ACM Int. Conf. MOBICOM2002, pp. 227-238, Atlanta, GA, USA, Sept. 2002.

6. Furey, E., Curran, K., McKevitt, P (2011e). Probabilistic Indoor Human Movement Modeling to Aid First Responders Journal of Ambient Intelligence and Humanized Computing. Ambient Intelligence Humanized Computing, Vol. 3, No. 2, DOI:10.1007/s12652-012-0112-4

7. Nafarieh, A., How, J. (2008) A Testbed for Localizing Wireless LAN Devices Using Received Signal Strength, Communication Networks and Services Research Conference, 2008. CNSR 2008. 6th Annual , vol., no., pp.481-487, 5-8 May 2008

8. Bardwell, J. (2002) Converting Signal Strength Percentage to dBm Value, WildPackets Inc.

9. Furey, E., Curran, K.. McKevitt, P (2011b). HABITS: A Bayesian Filter Approach to Indoor Tracking and Location, International Journal of Bio-Inspired Computation (IJBIC), Vol. 4, No. 1, pp: xx, ISSN (Print): 1758-0366, March 2012, InderScience

10. Carlin, S., Curran, K. (2014) An Active Low Cost Mesh Networking Indoor Tracking System. International Journal of Ambient Computing and Intelligence, Vol. 6, No. 1, January-March 2014, pp: 45-79, DOI: 10.4018/ijaci.2014010104

11. Curran, K., Furey, E. (2007) Pinpointing Users with Location Estimation Techniques and WiFi Hotspot Technology, International Journal of Network Management, Vol. 16, No. 5, Sept/Oct

12. Deak, G., Curran, K., Condell, J., Deak D. (2014) Detection of Multi-Occupancy using Device-free Passive Localisation (DfPL). IET Wireless Sensor Systems, Vol. 4, No. 2, pp: 1-8, June 2014, DOI: 10.1049/iet-wss.2013.0031

13. Mansell, G., Curran, K. (2016) Location Aware Tracking with Beacons. IPIN 2016 - The 7th Int Conference on Indoor Positioning \& Indoor Navigation, Madrid, Spain, 4-7 October 2016 
Please cite as: Kevin Curran, Gary Mansell, Jack Curran (2018) An loT Framework for detecting Movement within Indoor Environments. International Conference on Machine Learning for Networking (MLN'2018), Paris, France, November 27-29, 2018

\section{Acknowledgements}

This work was funded by the Royal Academy of Engineering under their Royal Academy of Engineering Senior Research Fellowship scheme. 